\title{
Structural optimisation of deployable scissor structures using new computational methods
}

\author{
L. Alegria Mira ${ }^{1}$, N. De Temmerman ${ }^{1} \&$ C. Preisinger ${ }^{2}$ \\ ${ }^{I}$ Department of Architectural Engineering, Vrije Universiteit Brussel, \\ Belgium \\ ${ }^{2}$ Bollinger-Grohmann-Schneider ZT mbH, Vienna, Austria
}

\begin{abstract}
In architectural engineering, deployable scissor structures are generally used for mobile and temporary applications. They are characterised by their dual functionality as either kinematic mechanisms (during deployment) or loadbearing skeletal structures (after deployment). It is crucial to realise that there is a direct and mutual relationship between the geometry, the kinematics and the structural response of the scissor system. Due to a relatively complex design process it can be highly beneficial to evaluate these structures at a pre-design stage in terms of their structural performance. In order to do so, new computational methods are introduced.

Karamba is a finite element plug-in for Grasshopper, fully embedded in the 3D modelling software Rhinoceros, which calculates interactively the response of three dimensional beam structures. The advantage of this new tool is the compatibility with the parametric environment of Grasshopper. These software tools are still in development, but already show their potential in terms of geometric modelling and structural optimisation.

In this research it is shown in which way these evolving computational methods can contribute to the design of deployable scissor structures. By using the proposed methodology of preliminary evaluation, the scissor structures are geometrically and structurally optimised at an early stage, thereby enhancing the overall design process and facilitating further detailed analysis.
\end{abstract}

Keywords: deployable structures, scissor structures, parametric design, kinematics, structural analysis, structural optimisation. 


\section{Introduction}

Deployable structures are characterised by their ability to transform and to adapt to evolving needs and changing environments. They are prefabricated space frames which can expand from a compact bundle of components into a largerspan, load-bearing structural shape (Figure 1 and 2). Because of this dynamic, kinematic property they offer significant advantages over conventional, static structures for a wide spectrum of applications ranging from temporary architectural structures to the aerospace industry [1-4]. Deployable structures were classified by Hanaor and Levy [5] according to their morphological and kinematic characteristics creating a set of distinct subgroups. This paper is concerned with one of those subgroups: pantographs or scissor structures. These have - besides being transportable - the great advantage of speed and ease of erection and dismantling, while offering a huge volume expansion and a high deployment reliability [6].

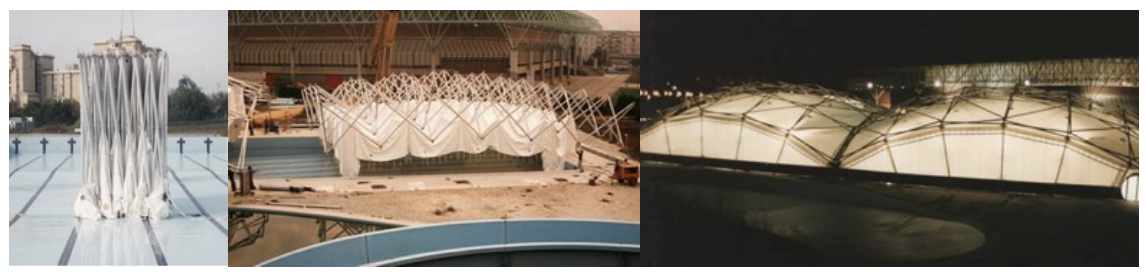

Figure 1: Deployable cover for swimming pool in Seville designed by Escrig and Sanchez [7].

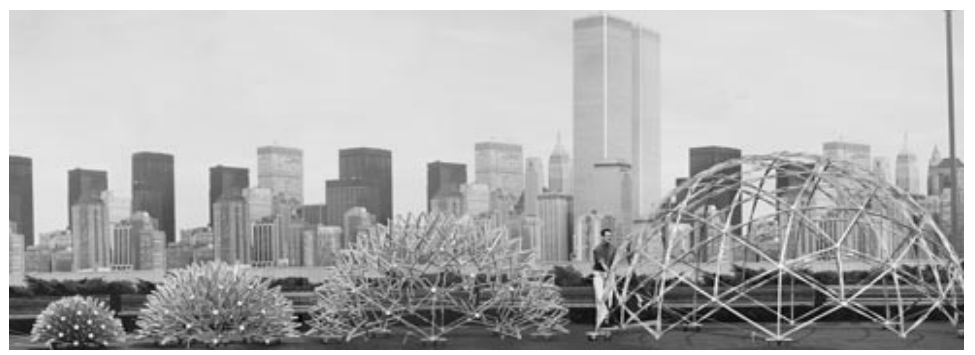

Figure 2: $\quad$ Expanding Geodesic Dome by Hoberman Associates Inc.(C) [8].

Despite the advantages scissor structures (or pantograph structures) can offer, few have successfully been realised. The design process is complex: a scissor structure requires a thorough understanding of the specific $2 \mathrm{D}$ and $3 \mathrm{D}$ configurations which will give rise to a fully deployable geometry. Moreover, structural implications must be considered. Flexure in the beams remains a major feature that detracts from structural efficiency $[5,9,10]$. The key element is that there is a direct and mutual relationship between the geometry, the kinematics and structural response of the scissor system [11]. 
Due to this complex design process it is beneficial to evaluate these structures at a pre-design stage according to their structural performance. For this, new computational methods are introduced in this paper. By using a methodology of preliminary evaluation, the scissor structures could be geometrically and structurally optimised at an early stage. This would enhance the overall design process, facilitate further detailed analysis and improve the performance of these structures which could result in more successful realisations.

\section{Scissor structures}

Scissor structures consist of units, also called scissor-like elements (SLE's). The scissor unit has revolute joints at all of its nodes, allowing a rotational degree of freedom about an axis perpendicular to the plane of the unit. At the centre node or intermediate hinge, the revolute joint connects the two beams comprising the scissor unit. By connecting such SLE's at their end nodes, a grid structure is formed, which can be transformed from a compact bundle of elements to a fully deployed configuration. Finally, by adding constraints, the mechanism goes from the deployment phase to the service phase, in which it can bear loads.

Depending on the location of the intermediate hinge and the shape of the beams, typically three main unit types can be distinguished: translational, polar and angulated units (Figure 3, 4 and 5). As the name suggests, translational units are mostly used in plane or curved scissor grids based on translational surfaces. The unit lines (dotted lines in the figures) are parallel and remain so during the deployment. Polar units are well-suited to form single-curvature grids such as barrel vaults, or the more basic scissor arch. With polar linkages, the unit lines intersect at an angle which varies continuously as the unit deploys. The angulated unit consists of members that have a kink angle, which gives rise to a unique property: the units can deploy radially, allowing the structure to 'grow' and 'shrink', while maintaining its overall shape.

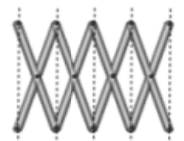

Figure 3: $\quad$ Translational linkage [11].

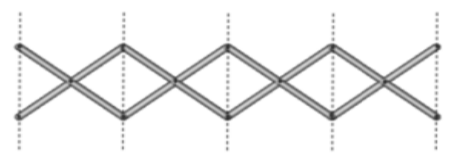

\section{Translational linkage [11].}
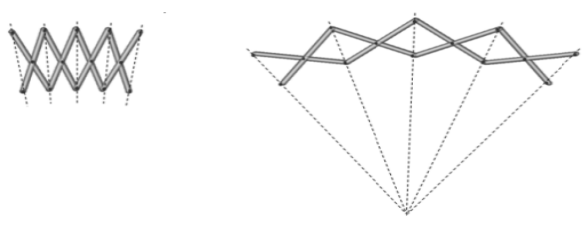

Figure 4: $\quad$ Polar linkage [11]. 

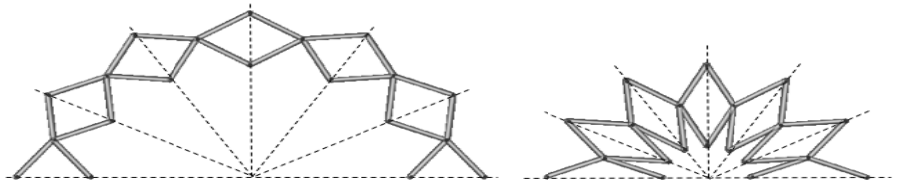

Figure 5: $\quad$ Angulated linkage [11].

It is important to understand that the chosen scissor type has a profound influence on the final shape of the space enclosure and its deployment behaviour.

In this paper it is investigated to what extent new digital tools can be used in order to optimise scissor structures. This is done by proposing a methodology and evaluating it in a preliminary way considering only simple $2 \mathrm{D}$ scissor linkages as illustrated in Figure 3, 4 and 5.

\section{Computational methods for structural assessment: Karamba}

\subsection{General}

The traditional way of composing geometries by means of CAD-programs concentrates on geometric objects like points, lines and surfaces which need to be placed manually by the user. This approach renders first results fast, but makes it time-consuming and error-prone to incorporate changes - which invariably arise in real world architectural projects. A more flexible method consists in identifying the relationships between the geometric objects that make up the drawing. Parametric modelling environments like the program Grasshopper (GH) [12] allow making these relationships explicit. They include them in an algorithmic structure. This results in a recipe for creating a specific version of a geometry based on a set of input parameters. This motivates the term 'parametric'.

In comparison to traditional approaches the parametric method normally proves more time intensive at the beginning of a geometric modelling task. However parametric environments allow users to interactively explore variants of geometries. This is a decisive advantage over traditional CAD-approaches: in large, complex building projects changes during all stages of development are frequent and thus necessitate a flexible design approach.

Karamba [13] is a finite element program which is optimised for use within the parametric geometric modelling environment Grasshopper [12] (GH). GH in turn operates as a plug-in for Rhinoceros [14] which is a computer aided design (CAD) package. Karamba comes not as a fully-fledged structural design tool for detailed engineering analysis. Instead it is meant to aid architects and engineers in principal design decisions during early project phases where flexibility in defining a static system scores over depth of detailing.

The basic working principles adopted in Karamba follow closely the foundations laid out in [15]. GH focuses on handling geometric objects. 
Transforming these into static models necessitates additional information: connectivity relations between geometric entities, material properties, support conditions and loads are defined via special user interface components. These lend their basic features from those contained within GH but are customised for their use in Karamba. Thus the look-and-feel properties of the Karamba user interface correspond largely to that of GH. This is an important fact regarding user acceptance. Besides components for describing the static properties of a structure Karamba also comprises so called process features [15]: they represent commands to the FE-solver and describe the solution procedure to be employed. One such object can e.g. stand for the determination of the displacement response under given loads, another one for example for calculating natural vibration modes.

An important aspect of Karamba is its bi-directionality with respect to calculation data: the model response attained through physical simulation can be fed back into the geometric model. This allows to set up automated design loops that rationalise designs by taking into account physical data: openings in a structural element can e.g. be concentrated in areas of low force density. GH contains feed-back components which facilitate the selection of sets of parameters which are optimal with respect to quantifiable but otherwise arbitrary criteria.

There have been several previous attempts at coupling parametric design tools and structural modelling software. Most of them lack bi-directionality of information transfer between geometric and finite element model. None of them allows truly interactive calculations. There are many factors that contribute to a time delay between changing the features of a model and display of static response:

(1) Traditional FE-programs are designed as stand-alone solutions and not as nodes within an integrated design system. Therefore they lack provisions for fast data exchange with external programs.

(2) Traditional FE-packages are meant to provide structural engineers with comprehensive data for detailing. The sort and extent of response data cannot be limited to the amount which would be useful in early design phase. The unwanted information wastes computational resources.

In Karamba the above described problems are avoided by implementing the calculation core as a dynamic link library which makes it easy and fast to access and control all aspects of the FE-model via scripting languages like $\mathrm{C \#}$ which is the language of choice within GH. An aspect of Karamba that adds to its speed of calculation is the fact that its capabilities are deliberately limited to that necessary in the early design phase: instead of e.g. employing isoparametric finite beam elements, hermitian elements are used. The latter are confined to linear elastic calculations of elements with straight axes. Yet the calculation of the element stiffness matrix can be done without the need for numeric integration and therefore very efficiently with respect to computation time. 


\subsection{Karamba for deployable scissor structures}

Scissor structures differ in an important aspect from other civil engineering structures: their elements connect in such a way that they form a moveable mechanism during the phase of erection. Afterwards the mechanism has to be locked in order to transform it into a load bearing structure. Due to the direct and mutual relation between geometry, kinematics and structural response, it is beneficial to evaluate these structures in a single modelling environment. Regarding the advantages, which are already explained in the previous section, this research introduces the modelling of scissor systems within the environment of Rhinoceros. Parametric modelling in Grasshopper facilitates the generation and adaptation of the structure's geometry, while also instantaneously assessing the kinematic behaviour (by the geometric conditions between the scissor elements) (Figure 6). Moreover the structural performance of the geometry can be directly traced with Karamba.

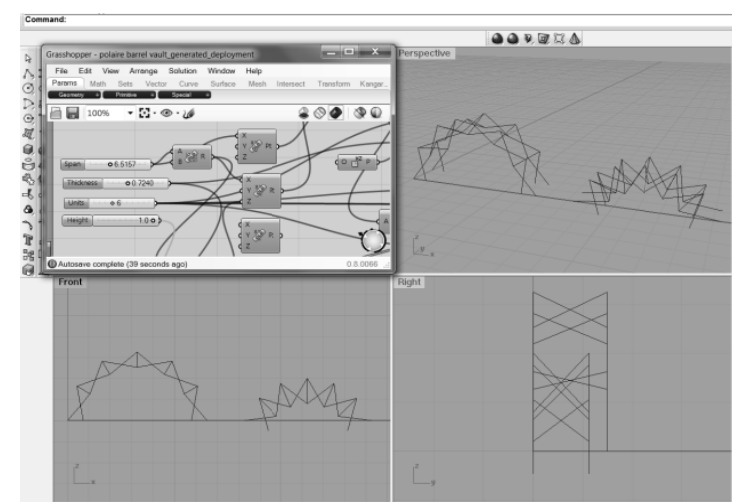

Figure 6: Screenshot of the parametric design plugin Grasshopper, generating the deployment of a scissor structure in the software Rhinoceros $3 \mathrm{D}$.

In order to be accessible to static calculations scissor structures have to be supported such that rigid body modes of displacement are eliminated. This can be done by controlling the scissor mechanism via imposed displacements at suitable points of the structure. Currently Karamba does not allow for large deflection analysis. In order to assess the behaviour of the structure at different stages during installation the corresponding geometric configurations have to be analysed.

In Karamba the rotational joints of the scissors are modelled as zero-length springs, which is an easy and effective method for a preliminary evaluation. The translational stiffness of the springs is set so that connecting nodes share the same coordinates and only the springs' rotational stiffness about the axis perpendicular to the plane of the scissor unit is zero. At this stage the structural properties of the hinges are not taken into account. This means that the non-zero 
stiffness values for the springs are set to a high value $\left(10^{11}-10^{13} \mathrm{kN}(\mathrm{m}) / \mathrm{m}\right)$, though limited to avoid a badly conditioned stiffness-matrix which would lead to inaccurate numerical results in the FE calculations.

In the following chapter the structural influence of different initial scissor configurations is determined with the use of Karamba. Based on the mentioned new computational methods an evaluation methodology of scissor structures is proposed leading to a geometrical and structural optimisation at an early stage enhancing the overall design process and further detailed analysis (Figure 7).

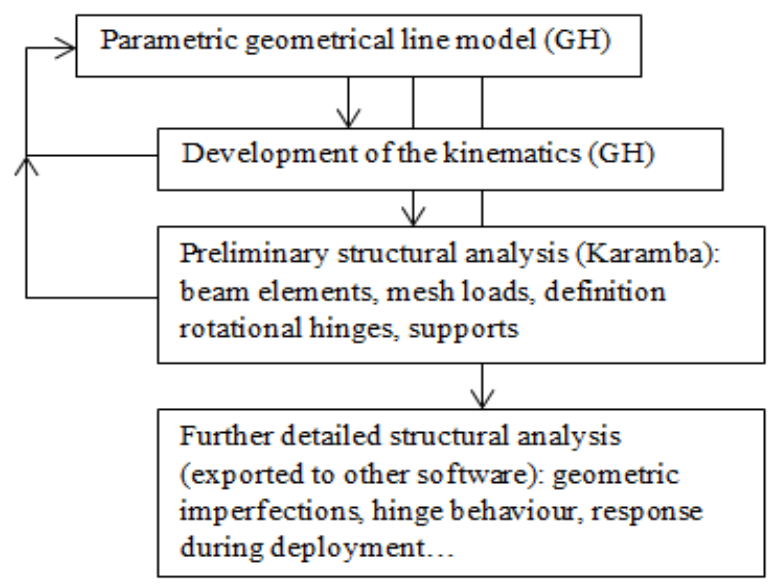

Figure 7: An evaluation methodology for deployable scissor structures based on parametric modelling.

\section{Structural analysis}

\subsection{Case studies}

In this section the computational methods, as explained in chapter 3 , are applied on a number of basic case studies of deployable scissor structures. The aim is to investigate if the proposed digital methods can be used for the structural evaluation of these types of structures in order to optimise them in an early design stage. The core of this paper is introducing the new digital tools in the research field of deployable scissor structures and clarifying how they are situated within and influence the proposed methodology (Figure 7). This is done by performing preliminary structural calculations on $2 \mathrm{D}$ scissor linkages. In reality $3 \mathrm{D}$ space enclosures are designed and these perform structurally different from 2D structures, but for this introductory paper the focus is put on a preliminary evaluation of the tools applied on 2D scissor cases.

The three main scissor units are considered: translational, polar and angulated (Figure 8). Each of them are examined in two differently curved arch shapes: a height-to-span ratio $(\mathrm{H} / \mathrm{S})$ of 0,25 and 0,5 respectively. Besides the H/S-ratio, the 
arches have the same geometrical properties to make a justified comparison: a span of $5 \mathrm{~m}, 5$ scissor units along the arch and a structural thickness of $0,5 \mathrm{~m}$ (Figure 9).
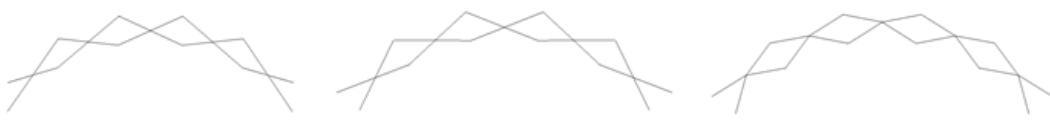

Figure 8: Examined scissor linkages with $\mathrm{H} / \mathrm{S}-$ ratio 0,25 - from left to right: translational, polar and angulated.
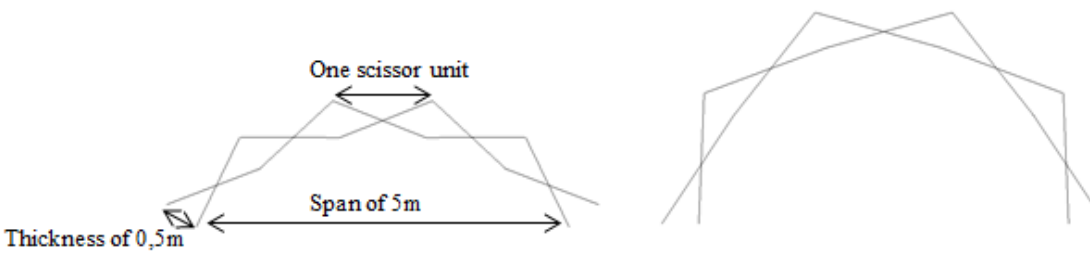

Figure 9: Polar linkage with $\mathrm{H} / \mathrm{S}=0,25$ (left) and $\mathrm{H} / \mathrm{S}=0,5$ (right).

The geometrical line models, built in a parametric way, are the input of the Karamba tool which calculates interactively the response of the scissor models as beam structures (all are circular tube profiles of steel grade S235 with an outer diameter of $10 \mathrm{~cm}$ and a wall-thickness of $0,3 \mathrm{~cm}$ ). The structural effects of parametric changes in the geometry of the line models are given in real-time with Karamba. Supports are set at the four free beam ends of the scissor arches to approximate how the structure is supported in reality as much as possible. A random mesh load of $1 \mathrm{kN}$ is applied on all the upper nodes. In Figure 10 a Karamba model is displayed which illustrates the stresses over the cross section - blue means tension stress, red compression.

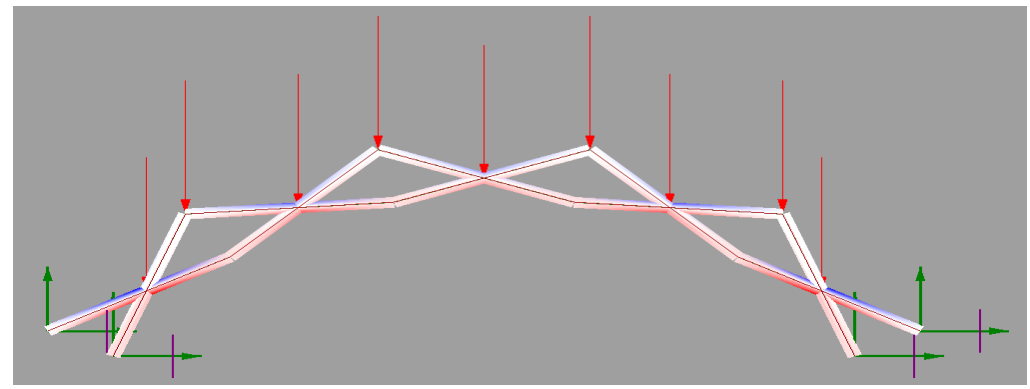

Figure 10: Karamba model view of an angulated scissor arch: indication of the loads, the supports and the stresses (blue is tension, red is compression and white represents an intermediate value). 
The numerical results will provide a preliminary judgment on the used scissor type and the examined architectural height.

\subsection{Numerical results}

Table 1 indicates the numerical output of Karamba for the different case studies. Figure 11 and 12 illustrate these results clearly for each height-to-span ratio. Different aspects of these results are evaluated in the following sections.

Table 1: The numerical results of the different case studies.

\begin{tabular}{|c|c|c|c|c|c|c|}
\hline & $\begin{array}{c}\text { Displ } \\
(\mathrm{m})\end{array}$ & $\begin{array}{c}\sigma_{\max } \\
(\mathrm{MPa})\end{array}$ & $\begin{array}{c}\mathrm{N}_{\max } \\
(\mathrm{kN})\end{array}$ & $\begin{array}{c}\mathrm{M}_{\max } \\
(\mathrm{kNm})\end{array}$ & $\begin{array}{c}\mathrm{V}_{\max } \\
(\mathrm{kN})\end{array}$ & $\begin{array}{c}\text { Member } \\
\text { Length }(\mathrm{m})\end{array}$ \\
\hline $\begin{array}{c}\text { Translational } \\
\mathrm{H} / \mathrm{S}=0,25\end{array}$ & 0,0024 & 61,7 & 7,69 & 1,24 & 9,06 & $0,89-1,53$ \\
\hline Translational H/S=0,5 & 0,0009 & 16,6 & 3,89 & 0,45 & 4,44 & $1,11-2,05$ \\
\hline Polar H/S=0,25 & 0,0032 & 57,2 & 4,17 & 1,22 & 6,56 & 1,34 \\
\hline Polar H/S=0,5 & 0,0004 & 7,1 & 1,36 & 0,17 & 2,95 & 1,76 \\
\hline Angulated H/S=0,25 & 0,0039 & 69,5 & 6,1 & 1,44 & 6,3 & 1,36 \\
\hline Angulated H/S=0,5 & 0,005 & 50,2 & 3,95 & 1,21 & 2,21 & 1,86 \\
\hline
\end{tabular}

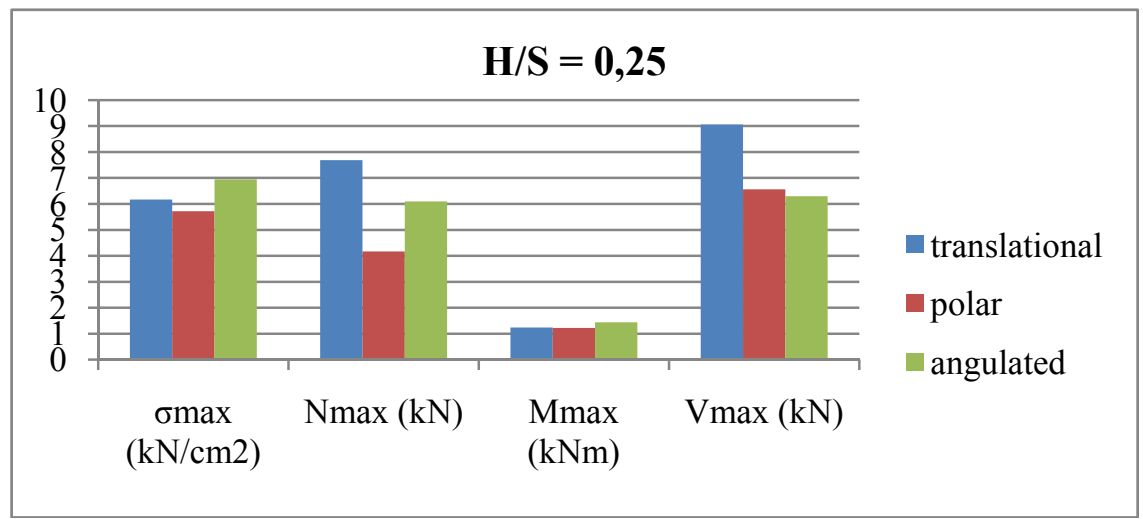

Figure 11: Comparison of the results between the different scissor units for $\mathrm{H} / \mathrm{S}=0,25$.

\subsubsection{Structural evaluation}

The comparison between Figure 11 and Figure 12 shows immediately that the geometry $\mathrm{H} / \mathrm{S}=0,5$ is structurally more efficient. The two translational scissor cases show the largest shear forces, while the angulated cases have the lowest shear forces. For the other parameters (displ $l_{\max }, \sigma_{\max }, \mathrm{N}_{\max }, \mathrm{M}_{\max }$ ), the two angulated cases have the highest values. The maximal displacement values (magnitude of $\mathrm{mm}$ ) are all negligible compared to the span of $5 \mathrm{~m}$. 


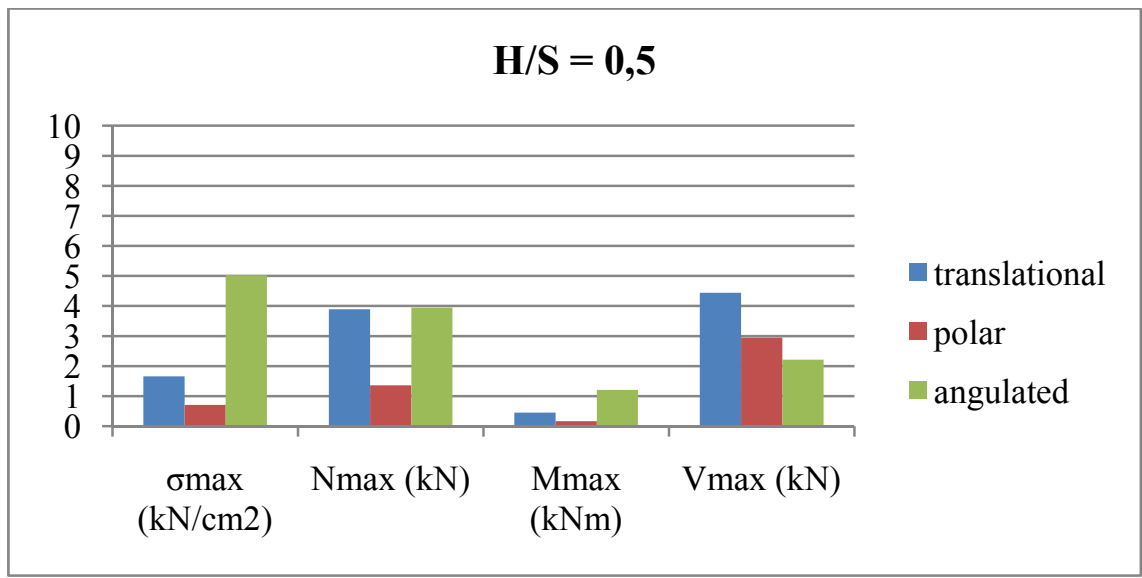

Figure 12: Comparison of the results between the different scissor units for $\mathrm{H} / \mathrm{S}=0,5$.

Generally it can be concluded from the numerical results that the polar configuration is the most efficient from a structural point of view. One has to bear in mind that this conclusion is applicable only for the investigated configuration (span of $5 \mathrm{~m}, 5$ scissor units and a thickness of $0,5 \mathrm{~m}$ ). To draw a more general conclusion other geometrical properties must be analysed (which with these digital methods could be done easily and fast).

The examination and analysis of the numerical output emphasised how efficient the proposed digital methods are for the evaluation of scissor structures. In real time the structural output is generated when the input parameters are changed. This means that a whole selection of different scissor geometries could be analysed extremely fast and different input parameters could be verified in terms of their structural influence. So, in this early design stage optimal scissor geometries can be chosen which are subsequently, at a later stage, optimised and analysed in greater detail.

\subsubsection{Manufacturing evaluation}

Besides the structural performance of a scissor geometry, the manufacturing properties can also influence the decision-making in the design process. Angulated scissor units consist of kinked beams which require a more complex manufacturing process than straight beams in case of translational and polar units.

Table 1 also gives the member length of each scissor beam per configuration. In case of the translational arches, the configuration is built up with 10 beams, each with a different length (ranging from $0,89 \mathrm{~m}$ to $1,53 \mathrm{~m}$ for $\mathrm{H} / \mathrm{S}=0,25$ and from $1,11 \mathrm{~m}$ to $2,05 \mathrm{~m}$ for $\mathrm{H} / \mathrm{S}=0,5$ ) leading to a more complex manufacturing process. This is not the case for angulated or polar scissors, in which all the members have the same length. 
So, looking from both a structural and a manufacturing point of view, the polar geometry is the most efficient for the investigated configuration.

Generally, the shorter the beam the less sensitive it is to buckling. The member lengths in table 1 show that the $\mathrm{H} / \mathrm{S}$ ratio of 0,25 leads to shorter beams. An important remark is that the buckling problem under axial loading of the scissor beams is not taken into account in the numerical analysis which defines the geometries with an H/S ratio of 0,5 as the structurally best performing. This must be included in future developments.

\subsubsection{Architectural evaluation}

The architectural evaluation of the configurations could also influence the design process to a certain extent, because the configurations form an envelope acting as a space enclosure. Here only a configuration with a single curvature is examined, which, through further research, must be extended to other 3D curved shapes.

For now, only 2D arches have been investigated, which differ in behaviour from the 3D barrel vaults they would represent in real life, but the architectural shape remains the same. Two configurations are investigated: a shallow curvature $(H / S=0,25)$ and an arch with a semi-circular shape $(H / S=0,5)$. These parameters immediately influence the usable enclosed space: the higher the H/S ratio, the more headroom is available inside the structure. So, also from an architectural and functional point of view the $\mathrm{H} / \mathrm{S}$ ratio of 0,5 is more beneficial.

\section{Conclusions}

The aim of this paper was to introduce new digital tools which are able to optimise the design process of deployable scissor structures. There is a direct relation between the geometry, the kinematics and the structural response of scissor systems which makes the design often very complex. In previous research generally the geometry, the kinematics and the structural analysis are developed and investigated as separate entities (with different tools or software packages). In this research an evaluation method (figure 7) is proposed with new digital tools which provide an optimisation loop in the early design stage and within a single software environment. Using parametric tools for the geometry design (like Grasshopper within Rhinoceros) which are linked in real time to a structural calculation method (like Karamba within Grasshopper) is a large step forward. This approach allows for the improvement of the design and realisation process of scissor structures, by taking the structural performance into account during the decision-making on geometrical design level.

Further research is needed on different levels. The evaluation methodology must be further optimised together with the developments and improvements of the digital tools. At present one configuration (span of $5 \mathrm{~m}, 5$ scissor units and a thickness of $0,5 \mathrm{~m}$ ) is investigated, but also other geometrical properties should be analysed, linking the input parameters for the design to their structural efficiency. An extension should be made to include 3D configurations, in addition to prototype tests comparing numerical and experimental results of the proposed methodology. 


\section{Acknowledgement}

The research presented in this paper is funded by a Ph.D. grant of the Agency for Innovation by Science and Technology (IWT) in Belgium.

\section{References}

[1] Gantes, C. J., Deployable Structures: Analysis and Design, WIT Press, 2001.

[2] Gantes, C. J., and Konitopoulou, E., Geometric design of arbitrarily curved bi-stable deployable arches with discrete joint size, International Journal of Solids and Structures, Vol. 41, 2004, pp. 5517-5540.

[3] Jensen, F. V., Concept for retractable roof structures, University of Cambridge, 2004.

[4] Rückert, G. C., Wandelbare hybride Konstruktionen Von der morphologischen Studie zum Prototyp, Technischen Hochschule Zürich, 2000.

[5] Hanaor, A., and Levy, R., Evaluations of Deployable Structures for Space Enclosures, International Journal of Space Structures, Vol. 16, No. 4, 2001, pp. 211-229.

[6] De Temmerman, N., Design and Analysis of Deployable Bar Structures for Mobile Architectural Applications, Vrije Universiteit Brussel, 2007.

[7] Performance S.L., http://www.performance-starbooks.com

[8] Hoberman Associates Inc.(C), http://www.hoberman.com

[9] Langbecker T., and Albermani F., Kinematic and non-linear analysis of foldable barrel vaults, Engineering Structures, Vol. 23, 2001, pp. 158-171.

[10] Richard Liew J.Y., Vu K.K., Krishnapillai A., Recent Development of Deployable Tension-Strut Structures, Advances in Structural Engineering, Vol. 11, No. 6, 2008, pp. 599-614.

[11] Alegria Mira, L., Design and Analysis of a Universal Scissor Component for Mobile Architectural Applications, Vrije Universiteit Brussel, 2010.

[12] Rutten, D., Grasshopper: generative modeling for Rhino (Computer software), www.grasshopper3d.com

[13] Preisinger, C., Karamba: parametric structural modeling (Computer software), www.karamba3d.com

[14] McNeel, R., RHINOCEROS: NURBS modeling for Windows (Computer software), www.rhino3d.com

[15] Coenders, J, Parametric and Associative Strategies for Engineering, IABSE Symposium Report of the IABSE Conference in Helsinki, 2008, pp. 15-22(8). 